\title{
DART, a BCA Code to Assess and Compare Primary Irradiation Damage in Nuclear Materials Submitted to Neutron and Ion Flux
}

\author{
Laurence LUNEVILLEE ${ }^{1,3}$, David SIMEONE ${ }^{2,3}$ \\ ${ }^{1}$ CEA-DEN-DM2S, Service d'Etudes des Réacteurs et de Mathématiques Appliquées, SERMA (Saclay, France) \\ ${ }^{2}$ CEA-DEN-DMN, Service de Recherches Métallurgiques Appliquées, SRMA (Saclay, France) \\ ${ }^{3}$ Laboratoire de Recherche Conventionné CARMEN (Paris \& Saclay, France)
}

When a material is subjected to a flux of high-energy particles, its constituent atoms can be knocked from their equilibrium positions with a wide range of energies, depending on the exact nature of the collision. The spectrum of damage energy, derived from the exact knowledge of the recoil spectra for each nuclear reaction occurring in the solid, constitutes a vital data set required for understanding how materials evolve under irradiation. The knowledge of such damage energy is relevant to compare the impact of different facilities on the structural behavior and relevant properties of materials.

The DART code [1] was developed for two distinct reasons: the first one was a correct determination of the Primary Knocked on Atoms (PKA) spectrum from reliable cross section data libraries and the second was a crude estimation of the damage energy induced by different irradiations. This last term can be a quick estimation of radiation damage produced in the same material by different nuclear plants and particle accelerators.

Based on the Binary Collision Approximation, this code allows computing the primary spectra produced by neutrons, ions and electrons as well as the damage energy deposited by these particles in a poly atomic material. It is then a tool to compare radiation damage induced in nuclear reactors as well as ion beam facilities.

Figure 1 displays the neutron spectra for different reactors: ITER (fusion reactor), HFIR (High Flux Isotope Reactor), IFMIF (International Fusion Materials Irradiation Facility) and PWR (Power Water Reactor).Two ion beam spectra (Ar $800 \mathrm{keV}$ and $\mathrm{Xe} 100 \mathrm{keV}$ ) are also plotted on this graph.

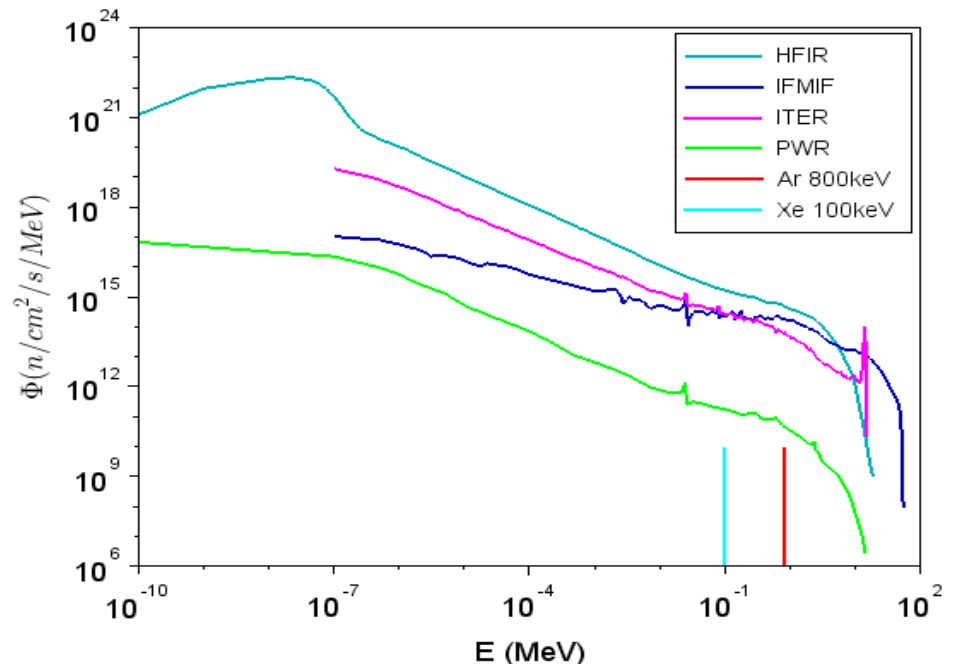

Fig. 1: Neutron spectra for different reactors and beam spectra.

DART calculations were performed to compare damage generated by these irradiations in pure iron. PKA spectra for the different irradiations are plotted on figure 2, showing the strong dependence of the incident spectrum on the energy distribution of recoils. 


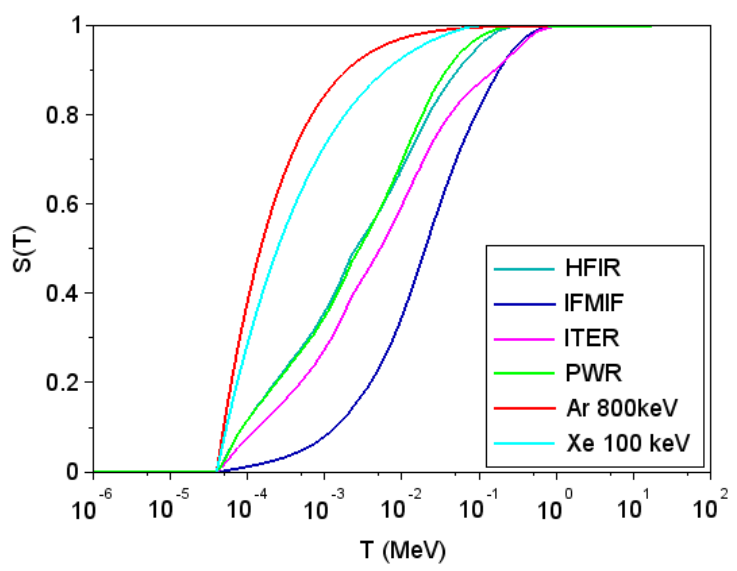

Fig. 2: Comparison of PKA spectra in pure iron for different irradiations using the same formalism for ion and neutron.

As DART allows defining a poly atomic material as a mixture of different isotopes (and not only of natural elements), we achieved the same calculations when adding to pure iron, $10 \%$ of a specific isotope of nickel, Ni-59. Figure 3 shows the increase of damage energy due to the presence of this element. Ni-59 increases the damage energy by more than an order of magnitude in HFIR reactor. This effect is related to Ni-59 reaction with thermal neutrons [2]. However, in fusion reactors, the low amount of thermal neutrons leads to a minimal impact on the damage energy due to the presence of Ni-59.

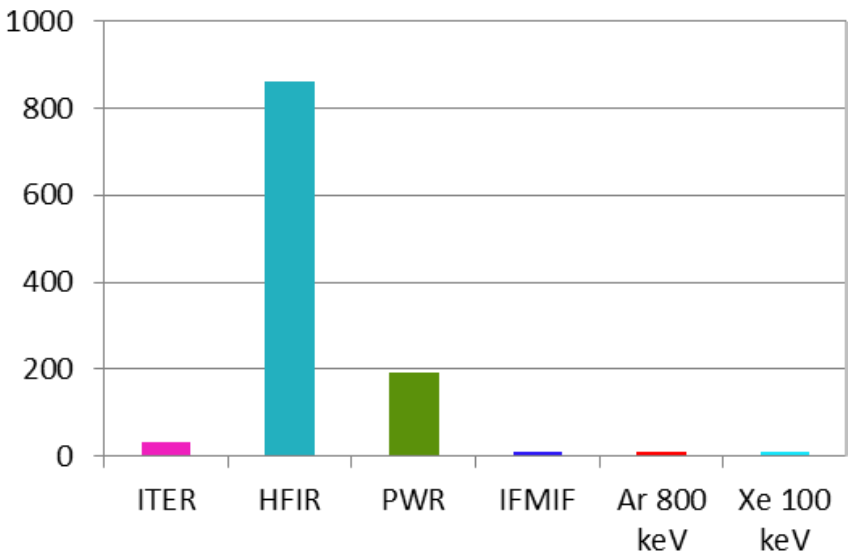

Fig. 3: Increase of the damage energy (in \%) when adding $10 \% \mathrm{Ni}-59$ in iron (effect of Ni-59 reaction with thermal neutrons).

It can also be pointed out that this result is strongly dependent on the Ni-59 neutron cross sections, showing the crucial role of the cross section data library.

\section{References}

[1] L. Luneville, D. Simeone, C. Jouanne, "Calculation of radiation damage induced by neutrons in compound materials", JNM 353, 89-100 (2006).

[2] F. A. Garner, L. R. Greenwood, E. R. Gilbert, M. Griffiths, 2009, "Impact of Ni-59 (n, $\alpha)$ and (n, p) Reactions on Dpa Rate, Heating Rate, Gas Generation and Stress Relaxation in LMR, LWR and CANDU reactors", Proc. 14 ${ }^{\text {th }}$ Intern. Conf. on Environmental Degradation of Materials in Nuclear Power Systems Water Reactors, Virginia Beach, Virginia, USA, August 23-27, 1344-1354 (2009). 
DART, A BCA CODE TO ASSESS AND

COMPARE PRIMARY IRRADIATION DAMAGE

IN NUCLEAR MATERIALS SUBMITTED TO

NEUTRON AND ION FLUX

L. Luneville, D. Simeone

ECP/CEA 


\section{Cea OUTLINE}

\section{MIN口S}

\section{Introduction \\ II Primary damage \\ III Results \\ IV Conclusion}




\section{INTRODUCTION}

\section{¿MINDS How to characterize damage in materials under irradiation?}

Interactions neutron-atoms defined with cross sections

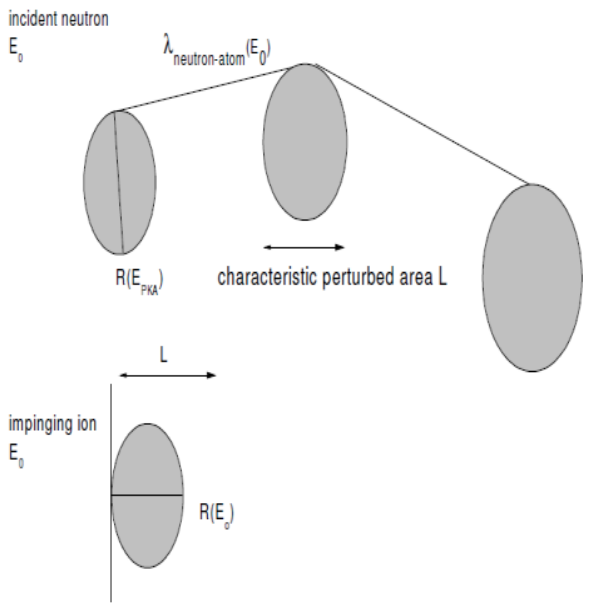

CEA - DEN

\section{Elastic interactions:}

$\sigma=$ few barns $\rightarrow$ macroscopic cross section $\Sigma=$ few $\mathrm{cm}$

The energy is given to the recoil atom : displacement cascades are well

$$
\text { separated }(>1 \mu \mathrm{m})
$$

Inelastic interactions:

* Nuclear reaction : emission of very energetic recoil atoms $(\mathrm{H}$, $\mathrm{He}$ ) but not only light atoms ex $\mathrm{UO}_{2}$ fission $\mathrm{U}^{235}$ emission of $\mathrm{Kr}$ ions $100 \mathrm{MeV}$

* Transmutation : modification of chemical species

some parts of the reactor (springs) are composed of inconel containing ${ }^{58} \mathrm{Ni}$ which transmutes in ${ }^{59} \mathrm{Ni}$ with high energy and then creates an increase of damage 


\section{INTRODUCTION}

\section{MINDS}

\section{Simulating a reactor irradiation with charged particles?}

\section{Ion irradiation}

* Simulate elastic collisions neutron-atoms

* Creation of displacement cascades

* Modified area under ion irradiation : a few hundred of nanometers

* Measurements near the surface (XR glancing incidence) far away from the implantation peak

* Irradiation with light ions : problem of deposited energy due to electronic loss

\section{Electron irradiation}

* No displacement cascades : isolated defects

* Measurements of displacement energies of an atom

* Measurements of the mobility of defects

*Impact of electronic loss 


\section{PRIMARY DAMAGE}

\section{¿MINDS Description of the displacement cascade induced by neutrons}
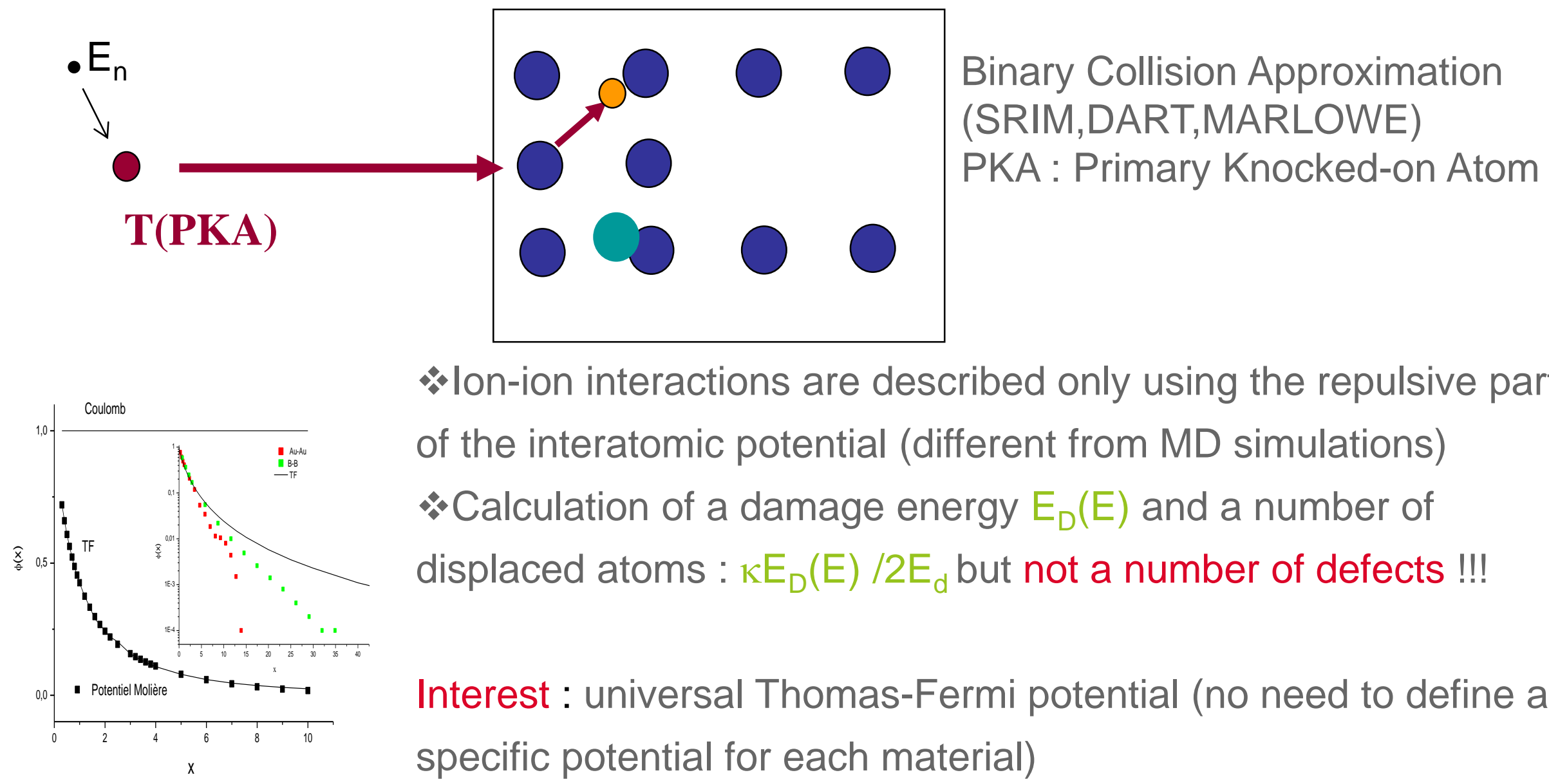

Ion-ion interactions are described only using the repulsive part of the interatomic potential (different from MD simulations) * Calculation of a damage energy $E_{D}(E)$ and a number of displaced atoms : $\kappa E_{D}(E) / 2 E_{d}$ but not a number of defects !!!

Interest : universal Thomas-Fermi potential (no need to define a specific potential for each material) Limitation : no defect calculation 


\section{PRIMARY DAMAGE}

\section{$\because$ MINDS}

Two elements to calculate the damage function (in the Binary Collision Approximation)

$\chi\left(E_{n}, T\right)$ : cross section neutron-atom : gives the energetic distribution $(T)$ of the recoil atoms displaced by a neutron of energy $E_{n}$

$E_{D}(T)$ : deposited energy by the ion : energy available for displacements i.e.when

substrating the electronic loss

Calculating the damage energy $E_{D}(T)$

DART : numerical resolution of Lindhard equations (polyatomic material)

electronic stopping power : the same as used by SRIM 2013 (based on measurements)

SRIM : Monte Carlo resolution of Lindhard equations (spatial description)

Marlowe : same as SRIM but taking into acount the cristalline structure of the material

Calculating the number of displaced atoms

$$
v(T)=\kappa E_{D}(T) / 2 E_{d} \text { where } E_{d} \text { is the displacement energy (NRT approximation) }
$$




\section{PRIMARY DAMAGE : DART}

\section{MINDS}

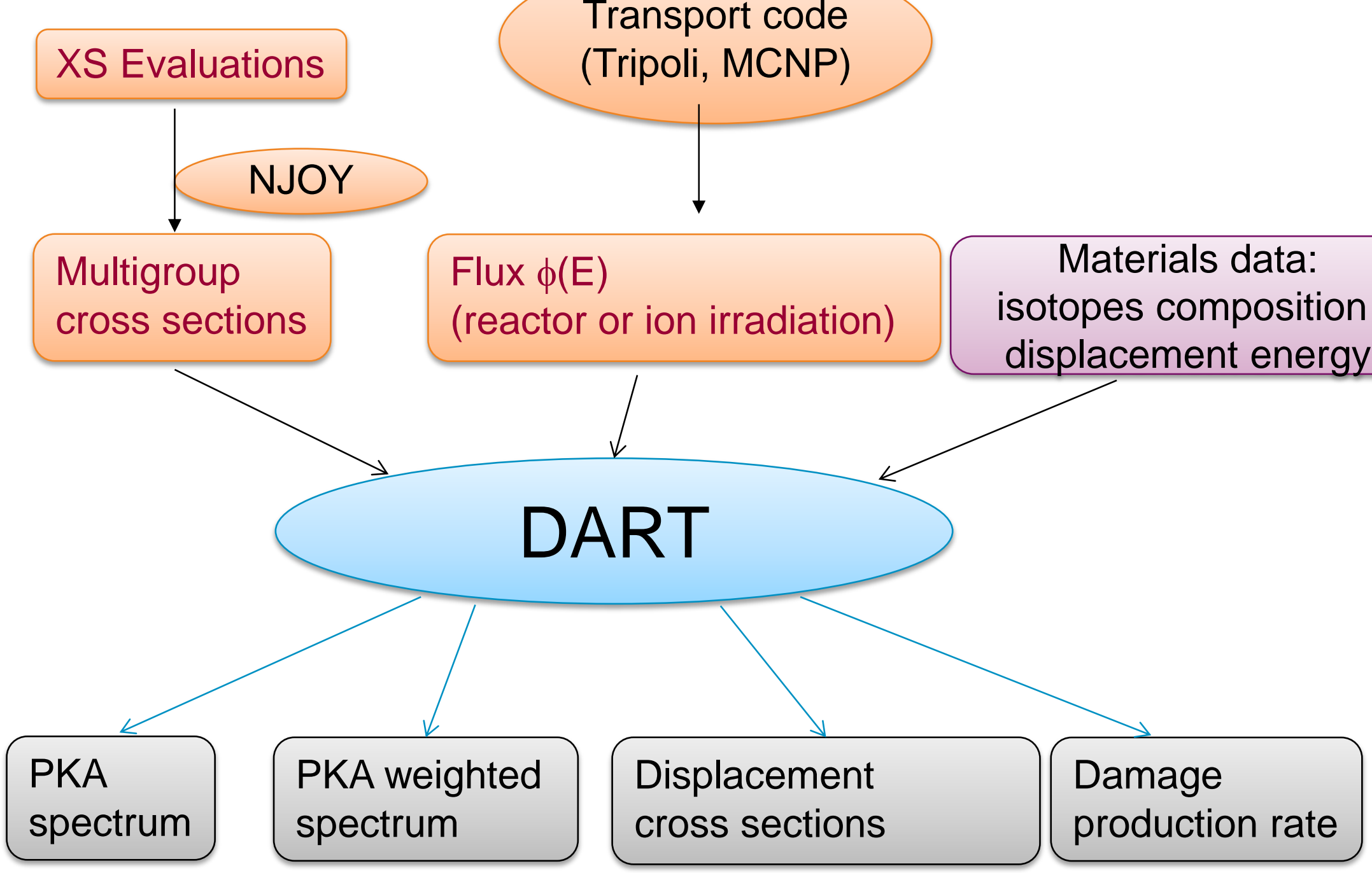




\section{PRIMARY DAMAGE : DART GRAPHIC USER INTERFACE}

\section{$\because$ MINDS}

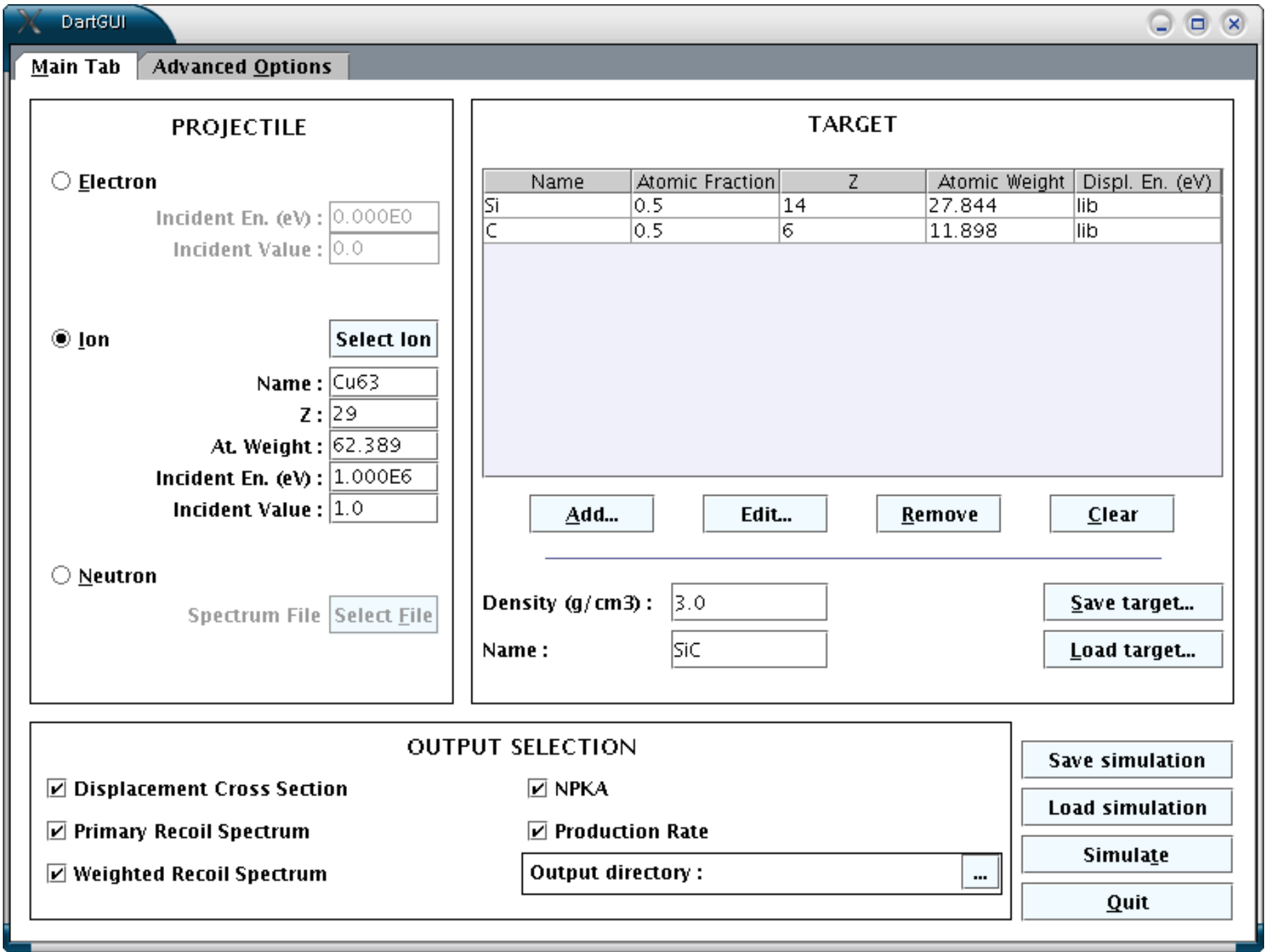




\section{RESULTS}

\section{¿MINDS How to compare different irradiations? Which estimators?}

\section{Damage production rate $(\mathrm{dpa} / \mathrm{s})$}

number of displaced atoms per second in the material

$$
R^{M a t}=\int \sigma_{d}^{M a t}(E) \phi(E) d E
$$

\begin{tabular}{|l|l|l|l|}
\hline Irradiation & $\begin{array}{l}\text { Total flux } \\
\left(\mathbf{n} / \mathrm{cm}^{2} / \mathbf{s}\right)\end{array}$ & $\begin{array}{l}\mathbf{R} \text { in Fe } \\
(\mathrm{d} p \mathrm{pa} / \mathrm{s})\end{array}$ & $\begin{array}{l}\text { Time to reach } \\
\mathbf{0 . 1} \mathrm{dpa} \text { in Fe }\end{array}$ \\
\hline HFIR & $510^{15}$ & $1.410^{-6}$ & $20 \mathrm{~h}$ \\
\hline IFMIF & $710^{14}$ & $1.210^{-6}$ & $23 \mathrm{~h}$ \\
\hline ITER & $410^{4}$ & $510^{-7}$ & $55 \mathrm{~h}$ \\
\hline PWR & $310^{11}$ & $9.810^{-11}$ & $280000 \mathrm{~h}$ \\
\hline Ar $800 \mathrm{keV}$ & $10^{12}$ & $4.910^{-4}$ & $200 \mathrm{~s}$ \\
\hline Xe 100keV & $10^{12}$ & $5.610^{-3}$ & $18 \mathrm{~s}$ \\
\hline
\end{tabular}

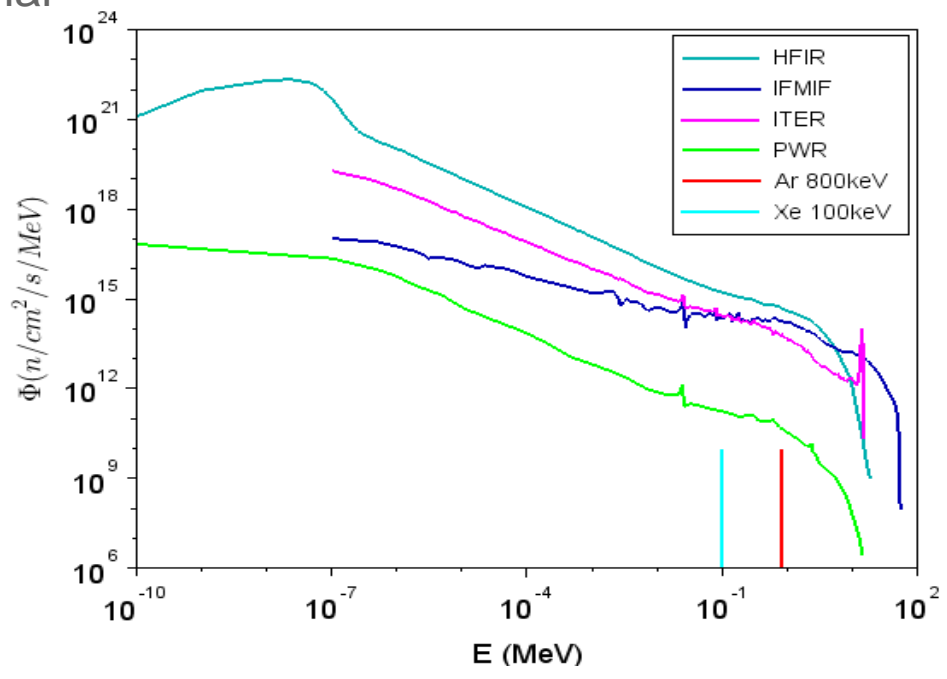

Flux for different reactors

Dpa rate is much higher for ion irradiation than in reactor. The time to obtain the same dpa must be reduced (factor 1000) in ion accelerators. 


\section{RESULTS}

\section{MINDS}

\section{How to compare different irradiations? Which estimators?}

PKA spectra in iron

PKA spectrum for monoatomic material

$\mathrm{S}(\mathrm{T})$ proportion of PKA having an energy $<\mathrm{T}$

* Strong dependence of the incident spectrum

* Same PKA spectrum means same distribution

of energy in the material

\section{PKA weighted spectrum for polyatomic material}

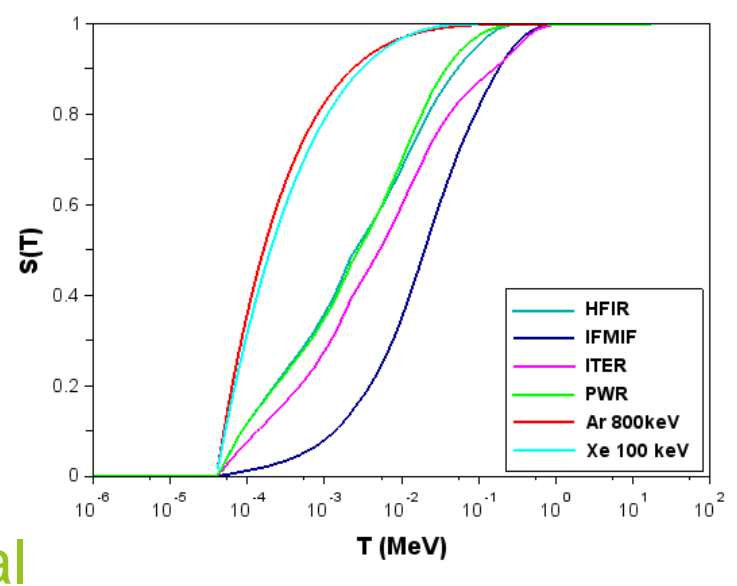

The energy given to the recoil gives different number of displacements in each sublattice of the material. The recoil energy is then weighted by the number of displaced atoms to define the PKA weighted spectrum

Comparison of PKA spectra for different irradiations

PKA weighted spectra in $\mathrm{Fe}-10 \%{ }^{58} \mathrm{Ni}$

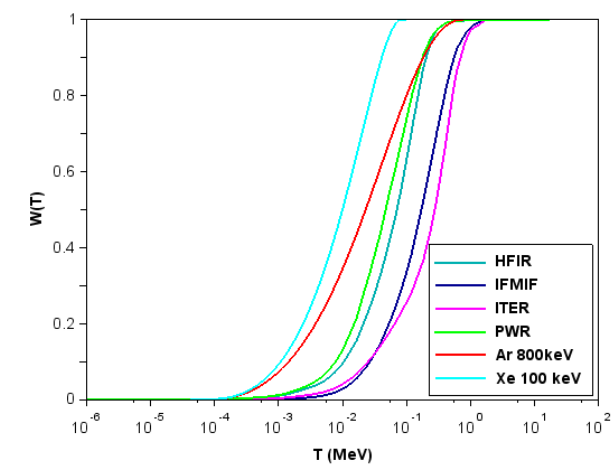




\section{RESULTS}

\section{$\because$ MINDS}

\section{Impact of XS evaluations (ENDF-BVII)}

Importance of Ni alloys (Inconel springs in Candu reactors)

${ }^{58} \mathrm{Ni}$ is the most abundant isotope in natural $\mathrm{Ni}$

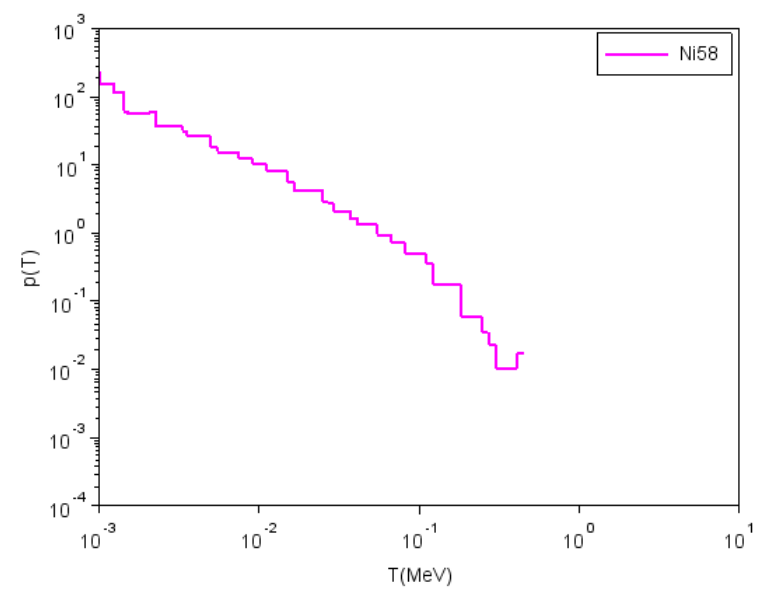

Energetic distribution of PKA after the collision between a neutron and a ${ }^{58} \mathrm{Ni}$ atom in the HFIR reactor 


\section{RESULTS}

\section{$\because$ MINDS}

\section{Impact of XS evaluations (ENDF-BVII)}

\section{Importance of Ni alloys (Inconel springs in Candu reactors)}

${ }^{58} \mathrm{Ni}$ is the most abundant isotope in natural $\mathrm{Ni}$

Under neutron irradiation a transmutation of ${ }^{58} \mathrm{Ni}$ occurs ${ }^{58} \mathrm{Ni}+\mathrm{n} \rightarrow{ }^{59} \mathrm{Ni}$

$\rightarrow$ apparition of ${ }^{59} \mathrm{Ni}$ not present in natural $\mathrm{Ni}$

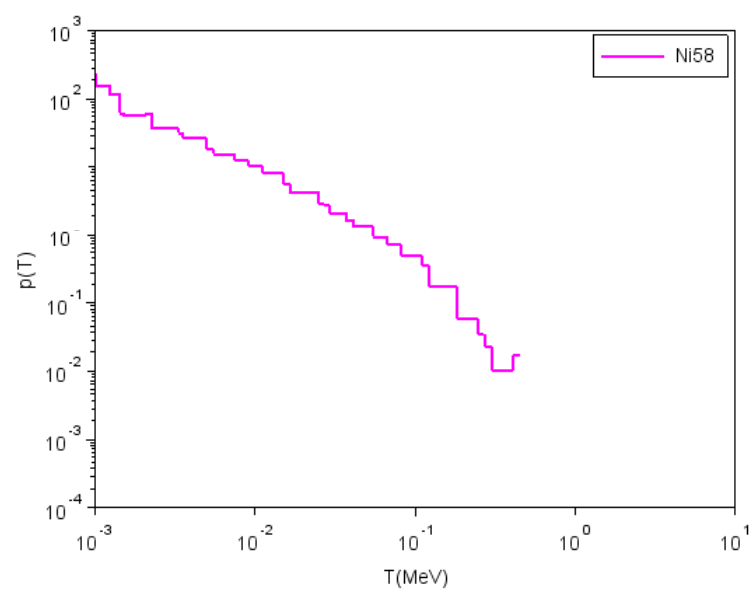

Energetic distribution of PKA after the collision between a neutron and a ${ }^{58} \mathrm{Ni}$ atom in the HFIR reactor

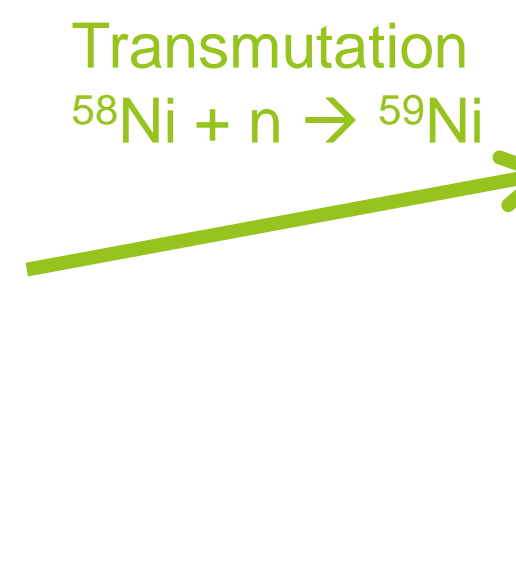
collision between a neutron and a ${ }^{59} \mathrm{Ni}$ atom in the HFIR reactor 


\section{RESULTS}

\section{$\because$ MINDS}

\section{Impact of XS evaluations (ENDF-BVII)}

\section{Importance of Ni alloys (Inconel springs in Candu reactors)}

The reaction of thermal neutrons on ${ }^{59} \mathrm{Ni}$ generates very energetic Fe ions (>500 keV) [Griffiths AECL Nucl. Rev 2 (2013)]

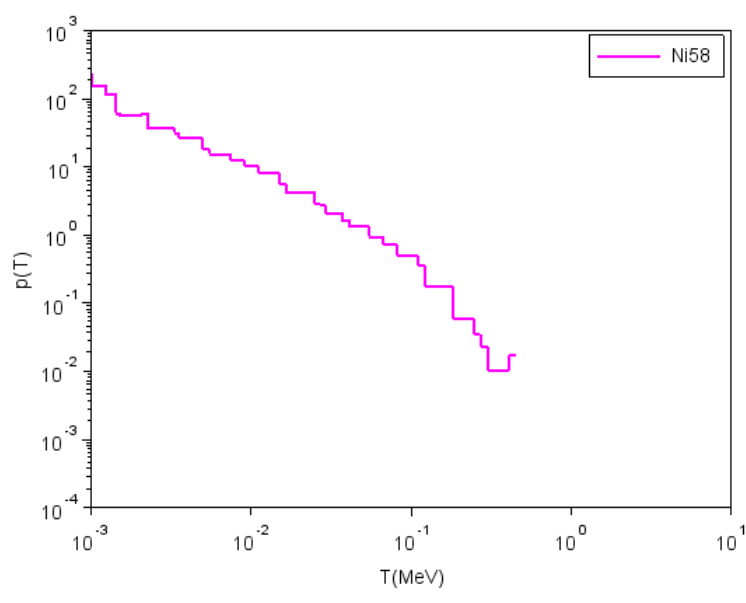

Energetic distribution of PKA after the collision between a neutron and a ${ }^{58} \mathrm{Ni}$ atom in the HFIR reactor

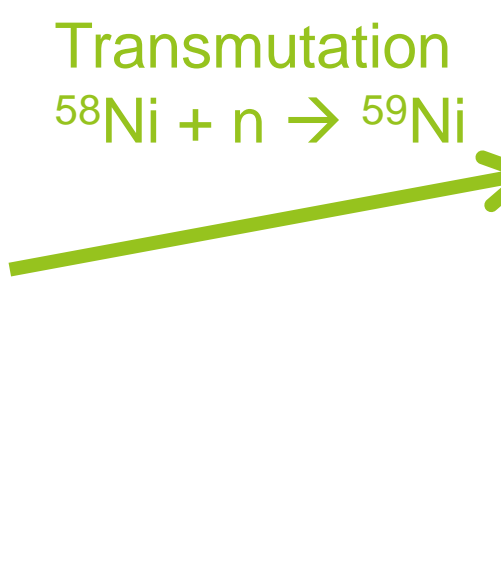
collision between a neutron and a ${ }^{59} \mathrm{Ni}$ atom in the HFIR reactor 


\section{RESULTS}

\section{MINDS}

\section{Impact of XS evaluations (ENDF-BVII)}

\section{Importance of Ni alloys (Inconel springs in Candu reactors)}

The reaction of thermal neutrons on ${ }^{59} \mathrm{Ni}$ generates very energetic Fe ions (>500 keV) [Griffiths AECL Nucl. Rev 2 (2013)]

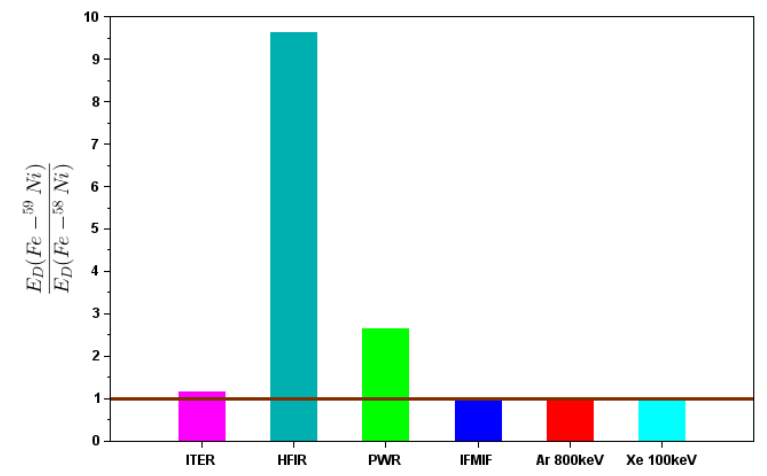

Ratio of the production rate between $\mathrm{Fe}-10 \%{ }^{59} \mathrm{Ni}$ and $\mathrm{Fe}-10 \% 58 \mathrm{Ni}$
Increase of the production rate

$1 \times 3$ in PWR $x 10$ in HFIR

$$
\begin{aligned}
& { }^{59} \mathrm{Ni}+\mathrm{n} \rightarrow{ }^{56} \mathrm{Fe}+\alpha \\
& 59 \mathrm{Ni}+\mathrm{n} \rightarrow{ }^{59} \mathrm{Co}+\mathrm{H}
\end{aligned}
$$

\section{Importance of}

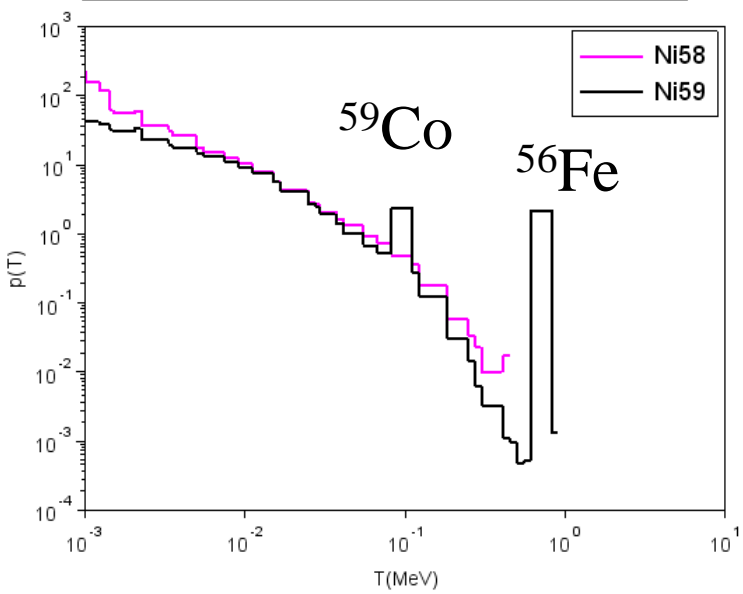

Energetic distribution of PKA after the collision between a neutron and a ${ }^{59} \mathrm{Ni}$ atom in the HFIR reactor

- good neutron cross sections

- calculations with isotopes and not natural elements 


\section{Cea CONCLUSION}

\section{¿MINas How to compare different irradiations?}

DART code in the BCA approximation allows calculating for ion and neutron irradiation :

* PKA spectra $\rightarrow$ choose ion and energy with the PKA spectrum as close as possible to the PKA spectrum with neutrons

\section{Choose ion}

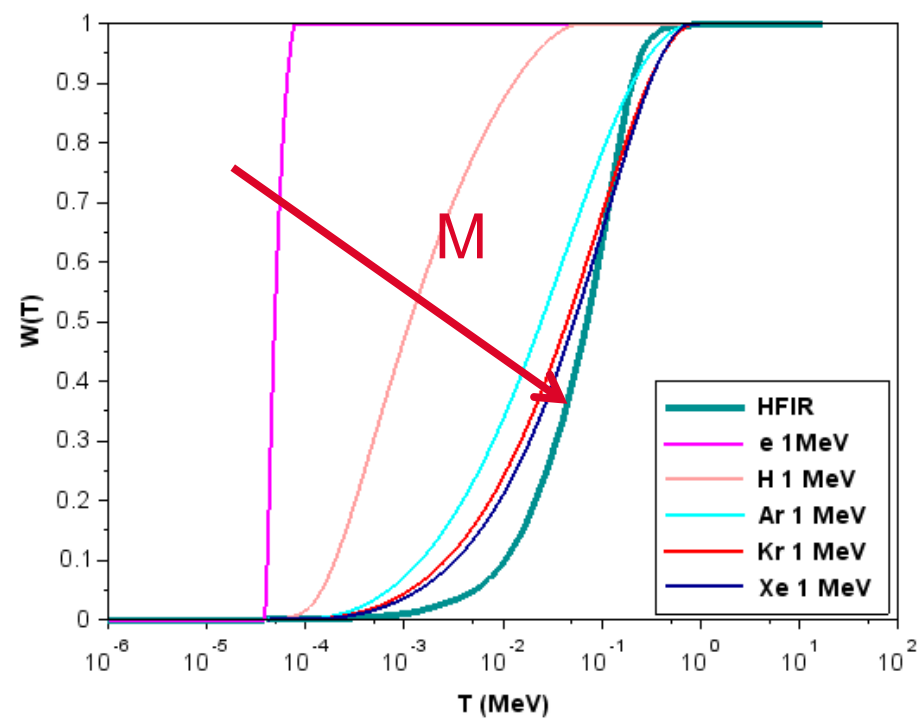

Choose energy

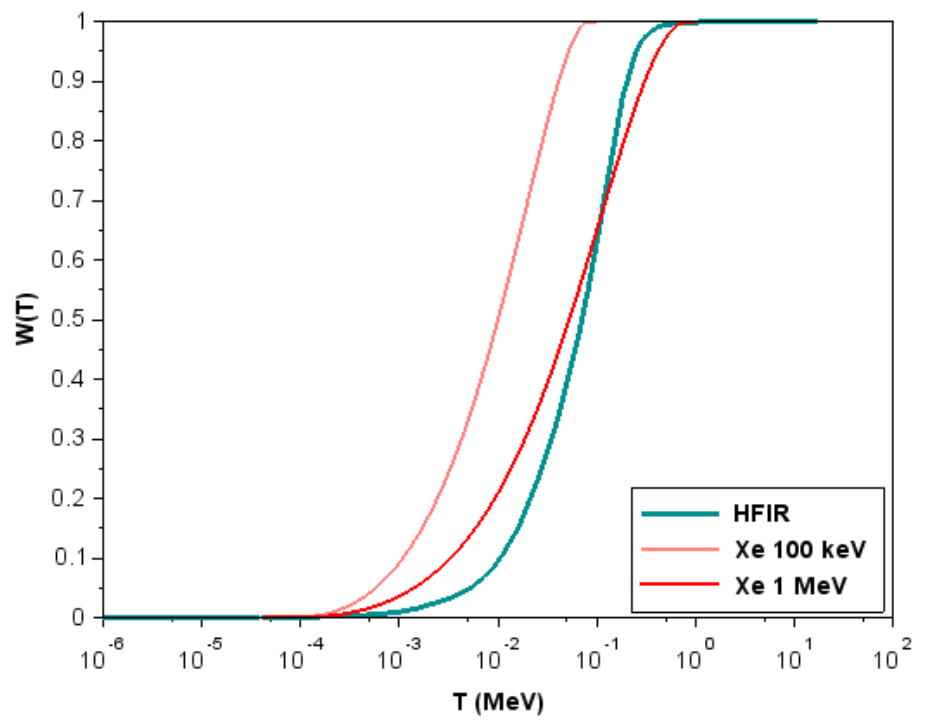

PKA weighted spectra in $\mathrm{Fe}-10 \%{ }^{58} \mathrm{Ni}$ 


\section{CONCLUSION}

\section{¿MINDS How to compare different irradiations?}

DART code in the BCA approximation allows calculating for ion and neutron irradiation :

* PKA spectra $\rightarrow$ choose ion and energy with the PKA spectrum as close as possible to the PKA spectrum with neutrons

$*$ Damage production rate (dpa/s) and damage (dpa) $\rightarrow$ choose time of ion irradiation to have the same dpa than with neutrons

* Validation with other codes

* Neutrons : SPECTER and HeATR (NJOY module)

* Ions : SRIM and MARLOWE 


\section{CONCLUSION}

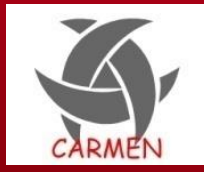

\section{MiNas How to compare different irradiations?}

DART code in the BCA approximation allows calculating for ion and neutron irradiation :

* PKA spectra $\rightarrow$ choose ion and energy with the PKA spectrum as close as possible to the PKA spectrum with neutrons

* Damage production rate (dpa/s) and damage (dpa) $\rightarrow$ choose time of ion irradiation to have the same dpa than with neutrons

(http://www.oecd-nea.org/tools/abstract/detail/nea-1885/)

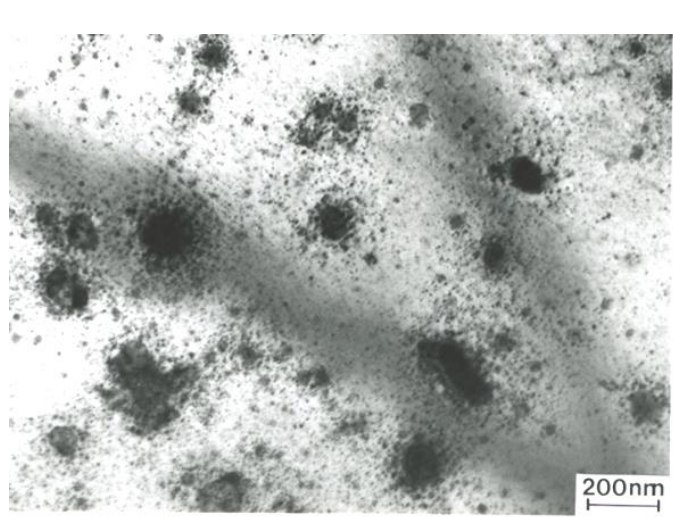

ODS irradiated in Phenix $750 \mathrm{~K}, 1.510^{-6} \mathrm{dpa} / \mathrm{s}, 30 \mathrm{dpa}$ (I. Monnet, Ph. D.)
CEA - DEN

\section{BUT}

BCA approximation (damage and not defects) $\rightarrow$ DM ?

* Even if the dpa is equal for ions and neutrons, the time of irradiation is 1000 times lower for ions $\rightarrow$ impact of time and flux ? * Simulation of primary damage (time of the displacement cascade $\left.10^{-12} \mathrm{~s}\right)$

Validation? Measurements of microstructure after several hours

\section{TO GO FURTHER}

Predict a microstructure : flux and T effects

Stationnary state induced by $1 \mathrm{MeV}$ Ar ions in $\mathrm{AgCu}$ at $450 \mathrm{~K}$ (Cu precipitates)

Phase Field simulation(G. Demange Ph D. $2^{\text {nd }}$ Int. MINOS Workshop - November 4-6, 2015, CEA - II

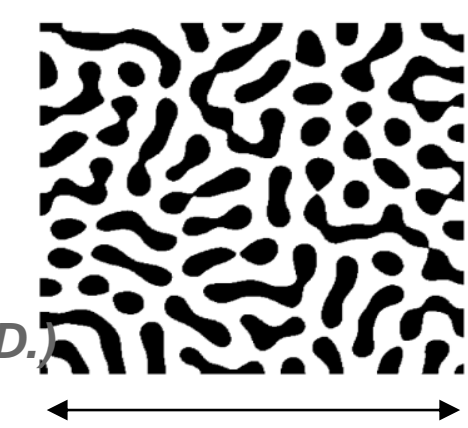

$100 \mathrm{~nm}$ 